\title{
SOME FIXED POINT RESULTS IN FUZZY CONE METRIC SPACES
}

\author{
N. Priyobarta ${ }^{1}$, Yumnam Rohen ${ }^{2}$, B.B. Upadhyay ${ }^{3}$ \\ ${ }^{1,2,3}$ Department of Mathematics \\ NIT Manipur \\ Imphal, 795001, INDIA
}

\begin{abstract}
In this manuscript, we prove some common fixed point theorems for occasionally weakly compatible mappings in fuzzy cone metric space.
\end{abstract}

AMS Subject Classification: 46S40, 54H25

Key Words: t-norm, fuzzy cone metric space, occasionally weakly compatible mappings

\section{Introduction}

The concept of fuzzy set was introduced by L.A. Zadeh [1] in 1965. After that many authors developed the theory of fuzzy sets and its applications. Kramosil and Michaleck [2] introduced fuzzy metric space in 1975. In 1999, R. Vasuki [3] proved fixed point theorems for $R$-weakly commutating mappings. In 2007 , Huang and Zhang [4] introduced the concept of cone metric space and proved some fixed point theorems for contractive mappings. In 2015, Tarkan Oner et al. [5] itroduced the concept of fuzzy cone metric space that generalized the corresponding notions of fuzzy metric space by George and Veeramani [6] and proved the fuzzy cone Banach contraction theorem. In recent past, several authors proved various fixed point theorems employing more generalized conditions.

Received:

Revised:

May 2, 2016

July 14, 2016

$\S$ Correspondence author (c) 2016 Academic Publications, Ltd. url: www.acadpubl.eu 
The aim of this paper is to prove some common fixed point theorems for occasionally weakly compatible mappings in fuzzy cone metric space.

\section{Preliminary Notes}

In this section, we start with some definitions.

Definition 2.1. [1] Let $X$ be any set. A fuzzy set $A$ in $X$ is a function with domain $X$ and values in $[0,1]$.

Definition 2.2. [5] Let $E$ be a real Banach space, $\theta$ the zero of $E$ and $P$ a subset of $E$. Then $P$ is called a cone if and only if

1. $P$ is closed, nonempty and $P \neq\{\theta\}$,

2. if $a, b \in R, a, b \geq 0$ and $x, y \in P$, then $a x+b y \in P$,

3. if both $x \in P$ and $-x \in P$, then $x=\theta$.

For a given cone $P$, a partial ordering $\preceq$ on $E$ with respect to $P$ is defined by $x \preceq y$ if and only if $y-x \in P$. The notation $x \prec y$ will stand for $x \preceq y$ and $x \neq y$, while $x \ll y$ will stand for $y-x \in \operatorname{int}(P)$. Throughout this paper, we assume that all cones have nonempty interior.

A cone $P$ is called normal if there exists a constant $K>0$ such that for all $t, s \in E, \theta \preceq t \preceq s$ implies $\|t\| \leq K\|s\|$ and the least positive number $K$ satisfying this property is called normal constant of $\mathrm{P}$ [5]. Rezapour and Hamlbarani [7] showed that there is no cone with normal constant $K<1$ and there exist cones of normal constant 1 , and cones of normal constant $M>K$ for each $K>1$.

Definition 2.3. [8] A binary operation $*:[0,1] \times[0,1] \rightarrow[0,1]$ is a continuous $t$-norm if $*$ satisfies the following conditions;

1. * is associative and commutative,

2. * is continuous,

3. $a * 1=a$ for all $a \in[0,1]$,

4. $a * b \leq c * d$ whenever $a \leq c$ and $b \leq d, a, b, c, d \in[0,1]$.

Example 2.4. $a * b=\min \{a, b\}$. 
Definition 2.5. [6] A 3-tuple $(X, M, *)$ is said to be a fuzzy metric space if $X$ is an arbitrary set, $*$ is a continuous $t$-norm and $M$ is a fuzzy set on $X^{2} \times(0, \infty)$ satisfying the following conditions;

FM1) $M(x, y, t)>0$,

FM2) $M(x, y, t)=1$ if and only if $x=y$,

FM3) $M(x, y, t)=M(y, x, t)$,

FM4) $M(x, y, t) * M(y, z, s) \leq M(x, z, t+s)$,

FM5) $M(x, y,):.(0, \infty) \rightarrow[0,1]$ is continuous, $x, y, z \in X$ and $t, s>0$.

Definition 2.6. [6] Let $(X, M, *)$ be a fuzzy metric space, $x \in X$ and $\left\{x_{n}\right\}$ be a sequence in $X$. Then

1. $\left\{x_{n}\right\}$ is said to converge to $x$ if for any $t>0$ and any $r \in(0,1)$ there exists a natural number $n_{0}$ such that $M\left(x_{n}, x, t\right)>1-r$ for all $n \geq n_{0}$. We denote this by $\lim _{n \rightarrow \infty} x_{n}=x$ or $x_{n} \rightarrow x$ as $n \rightarrow \infty$.

2. $\left\{x_{n}\right\}$ is said to be a Cauchy sequence if for any $r \in(0,1)$ and any $t>0$ there exists a natural number $n_{0}$ such that $M\left(x_{n}, x_{m}, t\right)>1-r$ for all $n, m \geq n_{0}$.

3. $(X, M, *)$ is said to be a complete metric space if every Cauchy sequence is convergent.

Definition 2.7. [5] A 3-tuple $(X, M, *)$ is said to be a fuzzy cone metric space if $P$ is a cone of $E, X$ is an arbitrary set, $*$ is a continuous $t$-norm and $M$ is a fuzzy set on $X^{2} \times \operatorname{int}(P)$ satisfying the following conditions;

For all $x, y, z \in X$ and $t, s \in \operatorname{int}(P)$ (that is $t \gg \theta, s \gg \theta$ )

FCM1) $M(x, y, t)>0$,

FCM2) $M(x, y, t)=1$ if and only if $x=y$,

FCM3) $M(x, y, t)=M(y, x, t)$,

$\mathrm{FCM} 4) M(x, y, t) * M(y, z, s) \leq M(x, z, t+s)$,

FCM5) $M(x, y,):. \operatorname{int}(P) \rightarrow[0,1]$ is continuous.

If $E=\mathbb{R}, P=[0, \infty)$ and $a * b=a b$, then every fuzzy metric spaces became a fuzzy cone metric spaces.

Example 2.8. [5] Let $E=\mathbb{R}^{2}$. Then $P=\left\{\left(k_{1}, k_{2}\right): k_{1}, k_{2} \geq 0\right\} \subset E$ is a normal cone with normal constant $K=1$. Let $X=\mathbb{R}, a * b=a b$ and $M: X^{2} \times \operatorname{int}(P) \rightarrow[0,1]$ defined by $M(x, y, t)=\frac{1}{e^{\frac{|x-y|}{\|t\|}}}$ for all $x, y \in X$ and $t \gg \theta$.

Definition 2.9. [5] Let $(X, M, *)$ be a fuzzy cone metric space, $x \in X$ and $\left\{x_{n}\right\}$ be a sequence in $X$. Then 
1. $\left\{x_{n}\right\}$ is said to converge to $x$ if for any $t \gg \theta$ and any $r \in(0,1)$ there exists a natural number $n_{0}$ such that $M\left(x_{n}, x, t\right)>1-r$ for all $n \geq n_{0}$. We denote this by $\lim _{n \rightarrow \infty} x_{n}=x$ or $x_{n} \rightarrow x$ as $n \rightarrow \infty$.

2. $\left\{x_{n}\right\}$ is said to be a Cauchy sequence if for any $0<\varepsilon<1$ and any $t \gg \theta$ there exists a natural number $n_{0}$ such that $M\left(x_{n}, x_{m}, t\right)>1-\varepsilon$ for all $n, m \geq n_{0}$.

3. $(X, M, *)$ is called complete if every Cauchy sequence is convergent.

Definition 2.10. [5] Let $(X, M, *)$ be a fuzzy cone metric space. A subset $A$ of $X$ is said to be FC-bounded if there exists $t \gg \theta$ and $r \in(0,1)$ such that $M(x, y, t)>1-r$ for all $x, y \in A$.

Definition 2.11. [9] Let $X$ be a nonempty set. An element $x \in X$ is called a common fixed point of mappings $F: X \times X \rightarrow X$ and $T: X \rightarrow X$ if $x=T(x)=F(x, x)$.

Definition 2.12. [9] Let $X$ be a nonempty set. The mappings $F: X \times$ $X \rightarrow X$ and $T: X \rightarrow X$ are called commutative if $T(F(x, y))=F(T(x), T(y))$ for all $x, y \in X$.

Definition 2.13. Let $X$ be a set, $F, T$ selfmaps of $X$. A point $x$ in $X$ is called a coincidence point of $F$ and $T$ if and only if $F(x)=T(x)$. We shall call $w=F(x)=T(x)$ a point of coincidence of $F$ and $T$.

Definition 2.14. A pair of maps $U$ and $V$ is called weakly compatible pair if they commute at coincidence points.

Definition 2.15. [10] Two self maps $F$ and $T$ of a set $X$ are occasionally weakly compatible if and only if there is a point $x$ in $X$ which is a coincidence point of $F$ and $T$ at which $F$ and $T$ commute.

A. Al-Thagafi and Naseer Shahzad [11] shown that occasionally weakly is weakly compatible but converse is not true.

Lemma 2.16. [12] Let $X$ be a set, $F, T$ occasionally weakly compatible self maps of $X$. If $F$ and $T$ have a unique point of coincidence, $w=F(x)=$ $T(x)$, then $w$ is the unique common fixed point of $F$ and $T$.

\section{Main Results}

We have the following theorems. 
Theorem 3.1. Let $(X, M, *)$ be a complete fuzzy cone metric space and let $A, B, U$ and $V$ be self-mappings of $X$. Let the pairs $\{A, U\}$ and $\{B, V\}$ be occasionally weakly compatible. If there exists $k \in(0,1)$ such that

$$
\begin{aligned}
M(A x, B y, k(t)) \geq & \min \{M(U(x), V(y), t), M(U(x), A(x), t) \\
& M(B(y), V(y), t), M(A(x), V(y), t) \\
& M(B(y), U(x), t)\}
\end{aligned}
$$

for all $x, y \in X$ and for all $t \gg \theta$, then there exists a unique point $w \in X$ such that $A(w)=U(w)=w$ and a unique point $z \in X$ such that $B(z)=V(z)=z$. Moreover, $z=w$, so that there is a unique common fixed point of $A, B, U$ and V.

Proof. Let the pairs $\{A, U\}$ and $\{B, V\}$ be occasionally weakly compatible, so there are points $x, y \in X$ such that $A(x)=U(x)$ and $B(y)=V(y)$. We claim that $A(x)=B(y)$. If not, by inequality (1)

$$
\begin{aligned}
M(A(x), B(y), k(t)) \geq & \min \{M(U(x), V(y), t), M(U(x), A(x), t), \\
& M(B(y), V(y), t), M(A(x), V(y), t), \\
& M(B(y), U(x), t)\} \\
= & \min \{M(A(x), B(y), t), M(A(x), A(x), t), \\
& M(B(y), B(y), t), M(A(x), B(y), t), \\
& M(B(y), A(x), t)\} \\
= & M(A(x), B(y), t) .
\end{aligned}
$$

Therefore $A(x)=B(y)$, i.e. $A(x)=U(x)=B(y)=V(y)$. Suppose that there is another point $z$ such that $A(z)=U(z)$ then by (1) we have $A(z)=U(z)=$ $B(y)=V(y)$, so $A(x)=A(z)$ and $w=A(x)=U(x)$ is the unique point of coincidence of $A$ and $U$. By Lemma 2.16, $w$ is the only common fixed point of $A$ and $U$. Similarly there is a unique point $z \in X$ such that $z=B(z)=V(z)$.

Assume that $w \neq z$. We have

$$
\begin{aligned}
M(w, z, k(t))= & M(A(w), B(z), k(t)) \\
\geq & \min \{M(U(w), V(z), t), M(U(w), A(z), t), \\
& M(B(z), V(z), t), M(A(w), V(z), t), M(B(z), U(w), t)\} \\
= & \min \{M(w, z, t), M(w, z, t), M(z, z, t), \\
& M(w, z, t), M(z, w, t)\}
\end{aligned}
$$




$$
=M(w, z, t) \text {. }
$$

Therefore we have $z=w$ by Lemma 2.16 and $z$ is a common fixed point of $A$, $B, U$ and $V$. The uniqueness of the fixed point holds from (1).

Theorem 3.2. Let $(X, M, *)$ be a complete fuzzy cone metric space and let $A, B, U$ and $V$ be self-mappings of $X$. Let the pairs $\{A, U\}$ and $\{B, V\}$ be occasionally weakly compatible. If there exists $k \in(0,1)$ such that

$$
\begin{aligned}
M(A(x), B(y), k(t)) \geq \quad & \phi[\min \{M(U(x), V(y), t), M(U(x), A(x), t), \\
& M(B(y), V(y), t), M(A(x), V(y), t), \\
& M(B(y), U(x), t)\}]
\end{aligned}
$$

for all $x, y \in X$ and $\phi:[0,1] \rightarrow[0,1]$ such that $\phi(t)>t$ for all $\theta \ll t<1$, then there exists a unique common fixed point of $A, B, U$ and $V$.

Proof. The proof follows from Theorem 3.1.

Theorem 3.3. Let $(X, M, *)$ be a complete fuzzy cone metric space and let $A, B, U$ and $V$ be self-mappings of $X$. Let the pairs $\{A, U\}$ and $\{B, V\}$ be occasionally weakly compatible. If there exists $k \in(0,1)$ such that

$$
\begin{aligned}
M(A(x), B(y), k(t)) \geq \quad & \phi(M(U(x), V(y), t), M(U(x), A(x), t), \\
& M(B(y), V(y), t), M(A(x), V(y), t), \\
& M(B(y), U(x), t))
\end{aligned}
$$

for all $x, y \in X$ and $\phi:[0,1]^{5} \rightarrow[0,1]$ such that $\phi(t, 1,1, t, t)>t$ for all $\theta \ll t<1$, then there exists a unique common fixed point of $A, B, U$ and $V$.

Proof. Let the pairs $\{A, U\}$ and $\{B, V\}$ are occasionally weakly compatible, there are points $x, y \in X$ such that $A(x)=U(x)$ and $B(y)=V(y)$. We claim that $A(x)=B(y)$. By inequality (3) we have

$$
\begin{aligned}
M(A(x), B(y), k(t)) \geq & \phi(M(U(x), V(y), t), M(U(x), A(x), t), \\
& M(B(y), V(y), t), M(A(x), V(y), t), \\
& M(B(y), U(x), t)) \\
= & \phi(M(A(x), B(y), t), M(A(x), A(x), t), \\
& M(B(y), B(y), t), M(A(x), B(y), t), \\
& M(B(y), A(x), t)) \\
= & \phi(M(A(x), B(y), t), 1,1, M(A(x), B(y), t),
\end{aligned}
$$




$$
\begin{aligned}
& M(B(y), A(x), t)) \\
>\quad & M(A(x), B(y), t)
\end{aligned}
$$

a contradiction, therefore $A(x)=B(y)$, i.e. $A(x)=U(x)=B(y)=V(y)$. Suppose that there is a another point $z$ such that $A(z)=U(z)$ then by (3) we have $A(z)=U(z)=B(y)=V(y)$, so $A(x)=A(z)$ and $w=A(x)=V(x)$ is the unique point of coincidence of $A$ and $U$. By Lemma 2.16, w is a unique common fixed point of $A$ and $U$. Similarly there is a unique point $z \in X$ such that $z=B(z)=V(z)$. Thus $z$ is a common fixed point of $A, B, U$ and $V$. The uniqueness of the fixed point holds from (3).

Theorem 3.4. Let $(X, M, *)$ be a complete fuzzy cone metric space and let $A, B, U$ and $V$ be self-mappings of $X$. Let the pairs $\{A, U\}$ and $\{B, V\}$ are occasionally weakly compatible. If there exists a point $k \in(0,1)$ for all $x, y \in X$ and $t \gg \theta$ satisfying

$$
\begin{aligned}
M(A(x), B(y), k(t)) \geq & M(U(x), V(y), t) * M(A(x), U(x), t) \\
& * M(B(y), V(y), t) * M(A(x), V(y), t)
\end{aligned}
$$

then there exists a unique common fixed point of $A, B, U$ and $V$.

Proof. Let the pairs $\{A, U\}$ and $\{B, V\}$ are occasionally weakly compatible, there are points $x, y \in X$ such that $A(x)=U(x)$ and $B(y)=V(y)$. We claim that $A(x)=B(y)$. By inequality (4), we have

$$
\begin{aligned}
M(A(x), B(y), k(t)) \geq & M(U(x), B(y), t) * M(A(x), U(x), t) \\
& * M(B y, B(y), t) * M(A(x), B(y), t) \\
= & M(A(x), B(y), t) * M(A(x), A(x), t) \\
& * M(B(y), B(y), t) * M(A(x), B(y), t) \\
\geq & M(A(x), B(y), t) * 1 * 1 * M(A(x), B(y), t) \\
\geq & M(A(x), B(y), t)
\end{aligned}
$$

Thus we have $A(x)=B(y)$, i.e. $A(x)=U(x)=B(y)=V(y)$. Suppose that there is a another point $z$ such that $A(z)=U(z)$ then by (4) we have $A(z)=U(z)=B(y)=V(y)$, so $A(x)=A(z)$ and $w=A(x)=U(x)$ is the unique point of coincidence of $A$ and $U$. Similarly there is a unique point $z \in X$ such that $z=B(z)=V(z)$. Thus $w$ is a common fixed point of $A, B, U$ and V. 
Corollary 3.5. Let $(X, M, *)$ be a complete fuzzy cone metric space and let $A, B, U$ and $V$ be self-mappings of $X$. Let the pairs $\{A, U\}$ and $\{B, V\}$ are occasionally weakly compatible. If there exists a point $k \in(0,1)$ for all $x, y \in X$ and $t \gg \theta$ satisfying

$$
\begin{aligned}
M(A(x), B(y), k(t)) \geq & M(U(x), V(y), t) * M(A(x), U(x), t) \\
& * M(B(y), V(y), t) * M(B(y), U(x), 2 t) \\
& * M(A(x), V(y), t)
\end{aligned}
$$

then there exists a unique common fixed point of $A, B, U$ and $V$.

Proof. We have

$$
\begin{aligned}
M(A(x), B(y), k(t)) \geq & M(U(x), V(y), t) * M(A(x), U(x), t) \\
& * M(B(y), V(y), t) * M(B(y), U(x), 2 t) \\
& * M(A(x), V(y), t) \\
\geq & M(U(x), V(y), t) * M(A(x), U(x), t) \\
& * M(B(y), V(y), t) * M(U(x), V(y), t) \\
& * M(T y, B(y), t) * M(A(x), V(y), t) \\
\geq & M(U(x), V(y), t) * M(A(x), U(x), t) \\
& * M(B(y), V(y), t) * M(A(x), V(y), t)
\end{aligned}
$$

and therefore from Theorem 3.4, $A, B, U$ and $V$ have a common fixed point.

Theorem 3.6. Let $(X, M, *)$ be a complete fuzzy cone metric space. Then continuous self mappings $U$ and $V$ of $X$ have a common fixed point in $X$ if and only if there exists a self mapping $A$ of $X$ such that the following conditions are satisfied

1. $A X \subset V X \cap U X$,

2. the pairs $\{A, U\}$ and $\{B, V\}$ are weakly compatible,

3. there exists a point $k \in(0,1)$ such that for every $x, y \in X$ and $t \gg \theta$

$$
\begin{aligned}
M(A(x), A(y), k(t)) \geq & M(U(x), V(y), t) * M(A(x), U(x), t) * \\
& M(A(y), V(y), t) * M(A(x), V(y), t)
\end{aligned}
$$

Then $A, U$ and $V$ have a unique common fixed point. 
Proof. Since compatible implies occasionally weakly compatible, the result follows from Theorem 3.4.

Theorem 3.7. Let $(X, M, *)$ be a complete fuzzy cone metric space and let $A$ and $B$ be self-mappings of $X$. Let the $A$ and $B$ are occasionally weakly compatible. If there exists a point $k \in(0,1)$ for all $x, y \in X$ and $t \gg \theta$

$$
\begin{aligned}
M(U(x), U(y), k(t)) \geq & \alpha M(A(x), A(y), t)+\beta \min \{M(A(x), A(y), t), \\
& M(U(x), A(x), t), M(U(y), A(y), t)\}
\end{aligned}
$$

for all $x, y \in X$, where $\alpha, \beta>0, \alpha+\beta>1$. Then $A$ and $U$ have a unique common fixed point.

Proof. Let the pairs $\{A, U\}$ be occasionally weakly compatible, so there is a point $x \in X$ such that $A(x)=U(x)$. Suppose that there exist another point $y \in X$ for which $A(y)=U(y)$. We claim that $U(x)=U(y)$. By inequality $(7)$ we have

$$
\begin{aligned}
M(U(x), U(y), k(t))= & \alpha M(A(x), A(y), t)+\beta \min \{M(A(x), A(y), t), \\
& M(U(x), A(x), t), M(U(y), A(y), t)\} \\
= & \alpha M(U(x), U(y), t)+\beta \min \{M(U(x), U(y), t), \\
& M(U(x), U(x), t), M(U(y), U(y), t)\} \\
= & (\alpha+\beta) M(U(x), U(y), t)
\end{aligned}
$$

a contradiction, since $(\alpha+\beta)>1$. Therefore $U(x)=U(y)$. Therefore $A(x)=$ $A(y)$ and $A(x)$ is unique. From Lemma 2.16, $A$ and $U$ have a unique fixed point.

\section{References}

[1] L. A. Zadeh, Fuzzy sets, Information and Computation, 8 (1965), 338-353.

[2] I. Kramosil and J. Michlek, Fuzzy metric and statistical metric spaces, Kybernetika, 11 (1975), 336-344.

[3] R. Vasuki, Common fixed points for R-weakly commuting maps in fuzzy metric spaces, Indian J. Pure Appl. Math. 30 (1999), 419-423.

[4] L. Huang and X. Zhang, Cone metric spaces and fixed point theorems of contractive mappings, J. Math. Anal. Appl., 332 (2007), 1468-1476.

[5] T. Oner, M.B. Kandemir and B. Tanay, Fuzzy cone metric spaces, J. Nonlinear Sci. and Appl. 8 (2015), 610-616.

[6] A. George and P. Veeramani, On some results in fuzzy metric spaces, Fuzzy Sets Syst., 64 (1994), 395-399. 
[7] S. Rezapour and R. Hamlbarani, Some notes on paper Cone metric spaces and fixed point theorems of contractive mappings, J. Math. Anal. Appl. 345 (2008), 719-724.

[8] B. Schweizer and A. Sklar, Statistical metric spaces, Pacific J. Maths., 10 (1960), 313-334.

[9] J. X. Fang, Common fixed point theorems of compatible and weakly compatible maps in Menger spaces, Nonlinear Analysis, Theory, Method and Applications, 71 (2009), 18331843.

[10] G. Jungck and B. E. Rhoades, Fixed Point for Set Valued functions without Continuity, Indian J. Pure Appl. Math., 29 (1998), 771-779.

[11] A. Al-Thagafi and Naseer Shahzad, Generalized I-Nonexpansive Selfmaps and Invariant Approximations, Acta Mathematica Sinica, English Series, 24 (2008), 867-876.

[12] G. Jungck and B. E. Rhoades, Fixed Point Theorems for Occasionally Weakly compatible Mappings, Fixed Point Theory, 7 (2006), 287-296. 